$\mathrm{J}$ o u r n a l of

Mathematics

and Applications

JMA No 41, pp 109-122 (2018)

\title{
Existence and Convergence Results for Caputo Fractional Volterra Integro-Differential Equations
}

\author{
Ahmed A. Hamoud*, M.Sh. Bani Issa, Kirtiwant P. Ghadle \\ and Mohammed Abdulghani
}

\begin{abstract}
In this article, homotopy analysis method is successfully applied to find the approximate solution of Caputo fractional Volterra integro-differential equation. The reliability of the method and reduction in the size of the computational work give this method a wider applicability. Also, the behavior of the solution can be formally determined by analytical approximate. Moreover, we proved the existence and convergence of the solution. Finally, an example is included to demonstrate the validity and applicability of the proposed technique.
\end{abstract}

AMS Subject Classification: 65H20, 26A33, 35C10.

Keywords and Phrases: Homotopy analysis method; Caputo fractional derivative; Volterra integro-differential equation; Approximate solution.

\section{Introduction}

In this paper, we consider Caputo fractional Volterra integro-differential equation of the form:

$$
{ }^{c} D^{\alpha} u(x)=g(x)+\int_{0}^{x} K(x, t) F(u(t)) d t,
$$

with the initial condition

$$
u^{(i)}(0)=\delta_{i}, \quad i=0,1,2, \cdots, n-1,
$$

COPYRIGHT (c) by Publishing House of Rzeszów University of Technology P.O. Box 85, 35-959 Rzeszów, Poland 
where ${ }^{c} D^{\alpha}$ is the Caputo's fractional derivative, $n-1<\alpha \leq n, n \in \mathbb{N}$ and $u: J \longrightarrow \mathbb{R}$, where $J=[0,1]$ is the continuous function which has to be determined, $g: J \longrightarrow \mathbb{R}$ and $K: J \times J \longrightarrow \mathbb{R}$, are continuous functions. $F: \mathbb{R} \longrightarrow \mathbb{R}$, is Lipschitz continuous function.

The fractional integro-differential equations have attracted much more interest of mathematicians and physicists which provides an efficiency for the description of many practical dynamical arising in engineering and scientific disciplines such as, physics, biology, electrochemistry, chemistry, economy, electromagnetic, control theory and viscoelasticity $[2,5,8,7,9,10,17,18,20]$. In recent years, many authors focus on the development of numerical and analytical techniques for fractional integrodifferential equations. For instance, we can remember the following works. An application of fractional derivatives was first given in 1823 by Abel [1] who applied the fractional calculus in the solution of an integral equation that arises in the formulation of the Tautochrone problem, Al-Samadi and Gumah [3] applied the homotopy analysis method for fractional SEIR epidemic model, Zurigat et al. [23] applied HAM for system of fractional integro-differential equations, Yang and Hou [20] applied the Laplace decomposition method to solve the fractional integro-differential equations, Mittal and Nigam [18] applied the Adomian decomposition method to approximate solutions for fractional integro-differential equations, and Ma and Huang [17] applied hybrid collocation method to study integro-differential equations of fractional order. Moreover, properties of the fractional integro-differential equations have been studied by several authors $[11,12,21,23]$. The homotopy analysis method (HAM) that was first proposed by Liao $[14,15,16]$, is implemented to derive analytic approximate solutions of fractional integro-differential equations (FIDEs) and convergence of HAM for this kind of equations is considered. Unlike all other analytical methods, HAM adjusts and controls the convergence region of the series solution via an auxiliary parameter $\hbar$.

The main objective of the present paper is to study the behavior of the solution that can be formally determined by analytical approximated method as the homotopy analysis method. Moreover, we proved the existence and convergence of the solution of the Caputo fractional Volterra integro-differential equation.

The rest of the paper is organized as follows: In Section 2, some preliminaries and basic definitions related to fractional calculus are recalled. In Section 3, homotopy analysis method is constructed for solving Caputo fractional Volterra integrodifferential equations. In Section 4, the existence and convergence of the solution have been proved. In Section 5, the analytical example is presented to illustrate the accuracy of this method. Finally, we will give a report on our paper and a brief conclusion is given in Section 6 .

\section{Preliminaries}

The mathematical definitions of fractional derivative and fractional integration are the subject of several different approaches. The most frequently used definitions of the fractional calculus involves the Riemann-Liouville fractional derivative, Caputo 
derivative $[13,19,22]$.

Definition 2.1. (Riemann-Liouville fractional integral). The Riemann-Liouville fractional integral of order $\alpha>0$ of a function $f$ is defined as

$$
\begin{aligned}
J^{\alpha} f(x) & =\frac{1}{\Gamma(\alpha)} \int_{0}^{x}(x-t)^{\alpha-1} f(t) d t, \quad x>0, \quad \alpha \in \mathbb{R}^{+}, \\
J^{0} f(x) & =f(x),
\end{aligned}
$$

where $\mathbb{R}^{+}$is the set of positive real numbers.

Definition 2.2. (Caputo fractional derivative). The fractional derivative of $f(x)$ in the Caputo sense is defined by

$$
\begin{aligned}
{ }^{c} D_{x}^{\alpha} f(x) & =J^{m-\alpha} D^{m} f(x) \\
& = \begin{cases}\frac{1}{\Gamma(m-\alpha)} \int_{0}^{x}(x-t)^{m-\alpha-1} \frac{d^{m} f(t)}{d t^{m}} d t, & m-1<\alpha<m, \\
\frac{d^{m} f(x)}{d x^{m}}, & \alpha=m, \quad m \in N,\end{cases}
\end{aligned}
$$

where the parameter $\alpha$ is the order of the derivative and is allowed to be real or even complex. In this paper, only real and positive $\alpha$ will be considered.

Hence, we have the following properties:

1. $J^{\alpha} J^{v} f=J^{\alpha+v} f, \quad \alpha, v>0$,

2. $J^{\alpha} x^{\beta}=\frac{\Gamma(\beta+1)}{\Gamma(\beta+\alpha+1)} x^{\beta+\alpha}$,

3. $J^{\alpha} D^{\alpha} f(x)=f(x)-\sum_{k=0}^{m-1} f^{(k)}\left(0^{+}\right) \frac{x^{k}}{k !}, \quad x>0, \quad m-1<\alpha \leq m$.

Definition 2.3. (Riemann-Liouville fractional derivative). The Riemann Liouville fractional derivative of order $\alpha>0$ is normally defined as

$$
D^{\alpha} f(x)=D^{m} J^{m-\alpha} f(x), \quad m-1<\alpha \leq m, \quad m \in \mathbb{N} .
$$

Theorem 2.4. [22] (Banach contraction principle). Let $(X, d)$ be a complete metric space, then each contraction mapping $T: X \longrightarrow X$ has a unique fixed point $x$ of $T$ in $X$ i.e. $T x=x$.

\section{Homotopy Analysis Method (HAM)}

Consider,

$$
N[u]=0,
$$

where $N$ is a nonlinear operator, $u(x)$ is unknown function and $x$ is an independent variable. Let $u_{0}(x)$ denote an initial guess of the exact solution $u(x), \hbar \neq 0$ an auxiliary parameter, $H_{1}(x) \neq 0$ an auxiliary function, and $L$ an auxiliary linear operator with the property $L[s(x)]=0$ when $s(x)=0$. Then using $q \in[0,1]$ as an 
embedding parameter, we can construct a homotopy when consider, $N[u]=0$, as follows $[4,6,14,15,21]$ :

$$
(1-q) L\left[\phi(x ; q)-u_{0}(x)\right]-q \hbar H_{1}(x) N[\phi(x ; q)]=\hat{H}\left[\phi(x ; q) ; u_{0}(x), H_{1}(x), \hbar, q\right] .
$$

It should be emphasized that we have great freedom to choose the initial guess $u_{0}(x)$, the auxiliary linear operator $L$, the non-zero auxiliary parameter $\hbar$, and the auxiliary function $H_{1}(x)$. Enforcing the homotopy Eq.(3.1) to be zero, i.e.,

$$
\hat{H}_{1}\left[\phi(x ; q) ; u_{0}(x), H_{1}(x), \hbar, q\right]=0,
$$

we have the so-called zero-order deformation equation

$$
(1-q) L\left[\phi(x ; q)-u_{0}(x)\right]=q \hbar H_{1}(x) N[\phi(x ; q)],
$$

when $q=0$, the zero-order deformation Eq.(3.3) becomes

$$
\phi(x ; 0)=u_{0}(x),
$$

and when $q=1$, since $\hbar \neq 0$ and $H_{1}(x) \neq 0$, the zero-order deformation Eq.(3.3) is equivalent to

$$
\phi(x ; 1)=u(x) .
$$

Thus, according to Eqs.(3.4) and (3.5), as the embedding parameter $q$ increases from 0 to $1, \phi(x ; q)$ varies continuously from the initial approximation $u_{0}(x)$ to the exact solution $u(x)$. Such a kind of continuous variation is called deformation in homotopy $[14,23]$. Due to Taylor's theorem, $\phi(x ; q)$ can be expanded in a power series of $q$ as follows

$$
\phi(x ; q)=u_{0}(x)+\sum_{m=1}^{\infty} u_{m}(x) q^{m}
$$

where

$$
u_{m}(x)=\left.\frac{1}{m !} \frac{\partial^{m} \phi(x ; q)}{\partial q^{m}}\right|_{q=0} .
$$

Let the initial guess $u_{0}(x)$, the auxiliary linear parameter $L$, the nonzero auxiliary parameter $\hbar$ and the auxiliary function $H_{1}(x)$ be properly chosen so that the power series (3.6) of $\phi(x ; q)$ converges at $q=1$, then, we have under these assumptions the solution series

$$
u(x)=\phi(x ; 1)=u_{0}(x)+\sum_{m=1}^{\infty} u_{m}(x) .
$$

From Eq.(3.6), we can write Eq.(3.3) as follows:

$$
\begin{aligned}
(1-q) L\left[\phi(x ; q)-u_{0}(x)\right] & =(1-q) L\left[\sum_{m=1}^{\infty} u_{m}(x) q^{m}\right] \\
& =q \hbar H_{1}(x) N[\phi(x ; q)],
\end{aligned}
$$


then

$$
L\left[\sum_{m=1}^{\infty} u_{m}(x) q^{m}\right]-q L\left[\sum_{m=1}^{\infty} u_{m}(x) q^{m}\right]=q \hbar H_{1}(x) N[\phi(x ; q)] .
$$

By differentiating Eq.(3.10) $m$ times with respect to $q$, we obtain

$$
\begin{aligned}
\left\{L\left[\sum_{m=1}^{\infty} u_{m}(x) q^{m}\right]-q L\left[\sum_{m=1}^{\infty} u_{m}(x) q^{m}\right]\right\}^{(m)} & =q \hbar H_{1}(x) N[\phi(x ; q)]^{(m)} \\
& =m ! L\left[u_{m}(x)-u_{m-1}(x)\right] \\
& =\left.\hbar H_{1}(x) m \frac{\partial^{m-1} N[\phi(x ; q)]}{\partial q^{m-1}}\right|_{q=0} .
\end{aligned}
$$

Therefore,

$$
L\left[u_{m}(x)-\chi_{m} u_{m-1}(x)\right]=\hbar H_{1}(x) \Re_{m}\left(\overrightarrow{u_{m-1}}(x)\right),
$$

where

$$
\Re_{m}\left(\overrightarrow{u_{m-1}}(x)\right)=\left.\frac{1}{(m-1) !} \frac{\partial^{m-1} N[\varphi(x ; q)]}{\partial q^{m-1}}\right|_{q=0},
$$

and

$$
\chi_{m}= \begin{cases}0, & m \leq 1 \\ 1, & m>1\end{cases}
$$

Note that the high-order deformation Eq.(3.11) is governing the linear operator $L$, and the term $\Re_{m}\left(\overrightarrow{u_{m-1}}(x)\right)$ can be expressed simply by Eq.(3.12) for any nonlinear operator $N$.

\section{HAM applied to fractional Volterra integro-differential equation}

We consider Caputo fractional Volterra integro-differential equation given by (1.1), with the initial condition (1.2). We can define

$$
N[\phi(x ; q)]={ }^{c} D^{\alpha} \phi(x ; q)-g(x)-\int_{0}^{x} K(x, t) F(\phi(t ; q)) d t .
$$

Now we construct the zero-order deformation equation

$$
(1-q)^{c} D^{\alpha}\left[\phi(x ; q)-u_{0}(x)\right]=q \hbar N[\phi(x ; q)],
$$

subject to the following initial conditions

$$
u_{0}(x)=\phi(0 ; q)=u_{0}=\sum_{k=0}^{n-1} \delta_{k} \frac{x^{k}}{k !},
$$


where $q \in[0,1]$ is the embedding parameter, $\hbar \neq 0$ is an auxiliary parameter, $u_{0}(x)$ is an initial guess of the solution $u(x)$ and $\phi(x ; q)$ is an unknown function on the independent variables $x$ and $q$. Also we suppose that

$$
{ }^{c} D^{\alpha}(C)=0,
$$

where $C$ is an integral constant. When the parameter $q$ increases from 0 to 1 , then the homotopy solution $\phi(x ; q)$ varies from $u_{0}(x)$ to solution $u(x)$ of the original equation (1.1). Using the parameter $q, \phi(x ; q)$ can be expanded in Taylor series as follows:

$$
\phi(x ; q)=u_{0}(x)+\sum_{m=1}^{\infty} u_{m}(x) q^{m}
$$

where $u_{m}(x)$ define as (3.7).

Assuming that the auxiliary parameter $\hbar$ is properly selected so that the above series is convergent when $q=1$, then the solution $u(x)$ can be given by

$$
u(x)=u_{0}(x)+\sum_{m=1}^{\infty} u_{m}(x) .
$$

Differentiating (3.13) and the initial condition (3.14) $m$ times with respect to $q$, then setting $q=0$, and finally dividing them by $m$ !, we get the $m^{\text {th }}$-order deformation equation

$$
{ }^{c} D^{\alpha}\left[u_{m}(x)-\chi_{m} u_{m-1}(x)\right]=\hbar \Re_{m}\left(\overrightarrow{u_{m-1}}(x)\right),
$$

subject to the following initial conditions,

$$
u_{m}(0)=0
$$

where

$$
\begin{aligned}
\Re_{m}\left(\overrightarrow{u_{m-1}}(x)\right) & =\left.\frac{1}{(m-1) !} \frac{\partial^{m-1} N[\phi(x ; q)]}{\partial q^{m-1}}\right|_{q=0} \\
& ={ }^{c} D^{\alpha} u_{m-1}(x)-\int_{0}^{x} K(x, t) F\left(u_{m-1}(t)\right) d t-\left(1-\chi_{m}\right) g(x),
\end{aligned}
$$

and

$$
\overrightarrow{u_{m}}=u_{0}, u_{1}, \cdots, u_{m}
$$

Applying the operator $J^{\alpha}$ to both sides of the linear $m$-order deformation (3.18)

$$
u_{m}(x)=\left(\chi_{m}+\hbar\right) u_{m-1}(x)-\hbar J^{\alpha}\left[\int_{0}^{x} K(x, t) F\left(u_{m-1}(t)\right) d t+\left(1-\chi_{m}\right) g(x)\right] .
$$




\section{Main Results}

In this section, we shall give an existence and uniqueness results of Eq. (1.1), with the initial condition (1.2) and prove it. Before starting and proving the main results, we introduce the following hypotheses:

(H1) There exists a constant $L_{F}>0$ such that, for any $u_{1}, u_{2} \in C(J, \mathbb{R})$

$$
\left|F\left(u_{1}(x)\right)-F\left(u_{2}(x)\right)\right| \leq L_{F}\left|u_{1}-u_{2}\right|
$$

(H2) There exists a function $K^{*} \in C\left(D, \mathbb{R}^{+}\right)$, the set of all positive function continuous on $D=\{(x, t) \in \mathbb{R} \times \mathbb{R}: 0 \leq t \leq x \leq 1\}$ such that

$$
K^{*}=\sup _{x \in[0,1]} \int_{0}^{x}|K(x, t)| d t<\infty .
$$

(H3) The function $g: J \rightarrow \mathbb{R}$ is continuous.

Lemma 4.1. If $u_{0}(x) \in C(J, \mathbb{R})$, then $u(x) \in C\left(J, \mathbb{R}^{+}\right)$is a solution of the problem (1.1) - (1.2) iff u satisfies

$$
\begin{aligned}
u(x)= & u_{0}+\frac{1}{\Gamma(\alpha)} \int_{0}^{x}(x-s)^{\alpha-1} g(s) d s \\
& +\frac{1}{\Gamma(\alpha)} \int_{0}^{x}(x-s)^{\alpha-1}\left(\int_{0}^{s} K(s, \tau) F(u(\tau)) d \tau\right) d s
\end{aligned}
$$

for $x \in J$, and $u_{0}=\sum_{k=0}^{n-1} \delta_{k} \frac{x^{k}}{k !}$.

Now, we will study the existence and uniqueness result of the solution based on the Banach contraction principle.

Theorem 4.2. Assume that (H1)-(H3) hold. If

$$
\left(\frac{K^{*} L_{F}}{\Gamma(\alpha+1)}\right)<1,
$$

then there exists a unique solution $u(x) \in C(J)$ to $(1.1)-(1.2)$.

Proof. By Lemma 4.1. we know that a function $u$ is a solution to (1.1) - (1.2) iff $u$ satisfies

$$
\begin{aligned}
u(x)= & u_{0}+\frac{1}{\Gamma(\alpha)} \int_{0}^{x}(x-s)^{\alpha-1} g(s) d s+\frac{1}{\Gamma(\alpha)} \int_{0}^{x}(x-s)^{\alpha-1} \\
& \times\left(\int_{0}^{s} K(s, \tau) F(u(\tau)) d \tau\right) d s
\end{aligned}
$$


Let the operator $T: C(J, \mathbb{R}) \rightarrow C(J, \mathbb{R})$ be defined by

$$
\begin{aligned}
(T u)(x)= & u_{0}+\frac{1}{\Gamma(\alpha)} \int_{0}^{x}(x-s)^{\alpha-1} g(s) d s \\
& +\frac{1}{\Gamma(\alpha)} \int_{0}^{x}(x-s)^{\alpha-1}\left(\int_{0}^{s} K(s, \tau) F(u(\tau)) d \tau\right) d s .
\end{aligned}
$$

Firstly, we prove that the operator $T$ is completely continuous. We can see that, if $u \in C(J, \mathbb{R})$ is a fixed point of $T$, then $u$ is a solution of $(1.1)-(1.2)$.

Now we prove $T$ has a fixed point $u$ in $C(J, \mathbb{R})$. For that, let $u_{1}, u_{2} \in C(J, \mathbb{R})$ and for any $x \in[0,1]$ such that

$$
\begin{aligned}
u_{1}(x)= & u_{0}+\frac{1}{\Gamma(\alpha)} \int_{0}^{x}(x-s)^{\alpha-1} g(s) d s \\
& +\frac{1}{\Gamma(\alpha)} \int_{0}^{x}(x-s)^{\alpha-1}\left(\int_{0}^{s} K(s, \tau) F\left(u_{1}(\tau)\right) d \tau\right) d s,
\end{aligned}
$$

and

$$
\begin{aligned}
u_{2}(x)= & u_{0}+\frac{1}{\Gamma(\alpha)} \int_{0}^{x}(x-s)^{\alpha-1} g(s) d s \\
& +\frac{1}{\Gamma(\alpha)} \int_{0}^{x}(x-s)^{\alpha-1}\left(\int_{0}^{s} K(s, \tau) F\left(u_{2}(\tau)\right) d \tau\right) d s .
\end{aligned}
$$

Consequently, we get

$$
\begin{aligned}
& \left|\left(T u_{1}\right)(x)-\left(T u_{2}\right)(x)\right| \\
\leq & \frac{1}{\Gamma(\alpha)} \int_{0}^{x}(x-s)^{\alpha-1}\left(\int_{0}^{s}|K(s, \tau)|\left|F\left(u_{1}(\tau)\right)-F\left(u_{2}(\tau)\right)\right| d \tau\right) d s \\
\leq & \frac{K^{*} L_{F}}{\Gamma(\alpha+1)}\left|u_{1}(x)-u_{2}(x)\right| \\
= & \left(\frac{K^{*} L_{F}}{\Gamma(\alpha+1)}\right)\left|u_{1}(x)-u_{2}(x)\right| .
\end{aligned}
$$

From the inequality (4.1) we have

$$
\left\|T u_{1}-T u_{2}\right\|_{\infty} \leq\left(\frac{K^{*} L_{F}}{\Gamma(\alpha+1)}\right)\left\|u_{1}-u_{2}\right\|_{\infty} .
$$

This means that $T$ is contraction map. By the Banach contraction principle, we can conclude that $T$ has a unique fixed point $u$ in $C(J, \mathbb{R})$.

Now, we will study the convergence theorem of the solutions based on the HAM.

Theorem 4.3. If the series solution $u(x)=\sum_{m=0}^{\infty} u_{m}(x)$ obtained by the m-order deformation is convergent, then it converges to the exact solution of the fractional Volterra integro-differential equation (1.1) - (1.2). 
Proof. We assume $\sum_{m=0}^{\infty} u_{m}(x)$ converge to $u(x)$ then

$$
\lim _{m \rightarrow \infty} u_{m}(x)=0 .
$$

We can write

$$
\begin{aligned}
\sum_{m=1}^{n}{ }^{c} D^{\alpha}\left[u_{m}(x)-\chi_{m} u_{m-1}(x)\right]= & { }^{c} D^{\alpha} u_{1}(x)+\left({ }^{c} D^{\alpha} u_{2}(x)-{ }^{c} D^{\alpha} u_{1}(x)\right) \\
& +\left({ }^{c} D^{\alpha} u_{3}(x)-{ }^{c} D^{\alpha} u_{2}(x)\right)+\ldots \\
& +\left({ }^{c} D^{\alpha} u_{n}(x)-{ }^{c} D^{\alpha} u_{n-1}(x)\right) \\
= & { }^{c} D^{\alpha} u_{n}(x) .
\end{aligned}
$$

Hence, from Eq.(4.2)

$$
\lim _{n \rightarrow \infty} u_{n}(x)=0 \text {. }
$$

So, using Eq.(4.3), we have

$$
\sum_{m=1}^{\infty}{ }^{c} D^{\alpha}\left[u_{m}(x)-\chi_{m} u_{m-1}(x)\right]=\sum_{m=1}^{\infty}\left[{ }^{c} D^{\alpha} u_{m}(x)-\chi_{m}{ }^{c} D^{\alpha} u_{m-1}(x)\right]=0 .
$$

Therefore from Eq.(4.3), we can obtain that

$$
\sum_{m=1}^{\infty}{ }^{c} D^{\alpha}\left[u_{m}(x)-\chi_{m} u_{m-1}(x)\right]=\hbar \sum_{m=1}^{\infty} \Re_{m-1}\left(\overrightarrow{u_{m-1}}(x)\right)=0 .
$$

Since $\hbar \neq 0$ and we have

$$
\sum_{m=1}^{\infty} \Re_{m-1}\left(\overrightarrow{u_{m-1}}(x)\right)=0 .
$$

By substituting $\Re_{m-1}\left(\overrightarrow{u_{m-1}}(x)\right)$ into the relation (4.4) and simplifying it, we have

$$
\begin{aligned}
\Re_{m-1}\left(\overrightarrow{u_{m-1}}(x)\right)= & \sum_{m=1}^{\infty}\left[{ }^{c} D^{\alpha} u_{m-1}(x)-\int_{0}^{x} K(x, t) F\left(u_{m-1}(t)\right) d t-\left(1-\chi_{m}\right) g(x)\right] \\
= & { }^{c} D^{\alpha}\left(\sum_{m=1}^{\infty} u_{m-1}(x)\right)-\int_{0}^{x} K(x, t)\left[\sum_{m=1}^{\infty} F\left(u_{m-1}(t)\right)\right] d t \\
& -\sum_{m=1}^{\infty}\left(1-\chi_{m}\right) g(x) \\
= & \left.{ }^{c} D^{\alpha} u(x)\right)-\int_{0}^{x} K(x, t) F(u(t)) d t-g(x) .
\end{aligned}
$$

From Eq.(4.4) and Eq.(4.5), we have

$$
{ }^{c} D^{\alpha} u(x)=g(x)+\int_{0}^{x} K(x, t) F(u(t)) d t,
$$

therefore, $u(x)$ must be the exact solution of Eq.(1.1) and the proof is complete. 


\section{Illustrative Example}

In this section, we present the analytical technique based on HAM to solve Caputo fractional Volterra integro-differential equations.

Example 1. Let us consider Caputo fractional Volterra integro-differential equation:

$$
{ }^{c} D^{0.5}[u(x)]=\frac{32-3 \sqrt{\pi}}{12 \sqrt{\pi}} x^{1.5}+\int_{0}^{x} \frac{t}{x^{2.5}} u(t) d t,
$$

with the initial condition

$$
u(0)=0
$$

From (3.13), (5.1) can be written as

$$
N[\phi(x ; q)]={ }^{c} D^{0.5} \phi(x ; q)-\frac{32-3 \sqrt{\pi}}{12 \sqrt{\pi}} x^{1.5}-\int_{0}^{x} \frac{t}{x^{2.5}} \phi(t ; q) d t .
$$

Now, using the $m^{\text {th }}$-order deformation equation (3.18) and initial conditions (3.19), and recursive equation $(3.20)$ we can write

$$
u_{m}(x)=\left(\chi_{m}+\hbar\right) u_{m-1}(x)-\hbar J^{0.5}\left[\left(1-\chi_{m}\right) \frac{32-3 \sqrt{\pi}}{12 \sqrt{\pi}} x^{1.5}+\int_{0}^{x} \frac{t}{x^{2.5}} u_{m-1}(t) d t\right] .
$$

Then,

$$
\begin{aligned}
u_{0}(x) & =0 \\
u_{1}(x) & =\hbar\left(\frac{3 \sqrt{\pi}}{32}-1\right) x^{2} \\
u_{2}(x) & =\hbar\left(1-\hbar\left(\frac{3 \sqrt{\pi}}{32}-1\right)\right)\left(\frac{3 \sqrt{\pi}}{32}-1\right) x^{2}, \\
u_{3}(x) & =\hbar\left(1-\hbar\left(\frac{3 \sqrt{\pi}}{32}-1\right)\right)^{2}\left(\frac{3 \sqrt{\pi}}{32}-1\right) x^{2}, \\
& \cdot \\
& \cdot \\
& \cdot \\
u_{n}(x) & =\hbar\left(1-\hbar\left(\frac{3 \sqrt{\pi}}{32}-1\right)\right)^{n-1}\left(\frac{3 \sqrt{\pi}}{32}-1\right) x^{2},
\end{aligned}
$$

thus the HAM series solution can be written as

$u_{m}(x)=\sum_{n=0}^{m} u_{n}(x)=\hbar\left(\frac{3 \sqrt{\pi}}{32}-1\right)\left[1+\left(1-\hbar\left(\frac{3 \sqrt{\pi}}{32}-1\right)\right)+\cdots+\left(1-\hbar\left(\frac{3 \sqrt{\pi}}{32}-1\right)\right)^{m-1}\right] x^{2}$. 
The exact solution of $(5.1)$ when $\frac{64}{3 \sqrt{\pi}-32}<\hbar<0$ is

$$
\begin{aligned}
u(x) & =\sum_{n=0}^{\infty} u_{n}(x) \\
& =\hbar\left(\frac{3 \sqrt{\pi}}{32}-1\right)\left[1+\left(1-\hbar\left(\frac{3 \sqrt{\pi}}{32}-1\right)\right)+\left(1-\hbar\left(\frac{3 \sqrt{\pi}}{32}-1\right)\right)^{2}+\cdots\right] x^{2} \\
& =\hbar\left(\frac{3 \sqrt{\pi}}{32}-1\right)\left(\frac{1}{1-\left(1-\hbar\left(\frac{3 \sqrt{\pi}}{32}-1\right)\right)}\right) x^{2}=x^{2}
\end{aligned}
$$

\section{Conclusions}

Homotopy analysis method is successfully applied to derive approximate analytical solutions for fractional Volterra integro-differential equations. Also, we proved the existence and convergence of the solution. Moreover, the obtained results show that we can control of the convergence district of homotopy analysis technique by control the auxiliary parameter $\hbar$. The convergence theorem and the illustrative example establish the precision and efficiency of the proposed technique.

\section{Acknowledgements}

The authors present their very grateful thanks to the editor and anonymous referees for their valuable suggestions and comments on improving this paper.

\section{References}

[1] N. Abel, Solution de quelques problemes a laide dintegrales definites, Christiania Grondahl, Norway (1881) 16-18.

[2] S. Alkan, V. Hatipoglu, Approximate solutions of Volterra-Fredholm integrodifferential equations of fractional order, Tbilisi Mathematical Journal 10 (2) (2017) 1-13.

[3] M. AL-Smadi, G. Gumah, On the homotopy analysis method for fractional SEIR epidemic model, Research J. Appl. Sci. Engrg. Technol. 7 (18) (2014) 3809-3820.

[4] S. Behzadi, S. Abbasbandy, T. Allahviranloo, A. Yildirim, Application of homotopy analysis method for solving a class of nonlinear Volterra-Fredholm integrodifferential equations, J. Appl. Anal. Comput. 2 (2) (2012) 127-136.

[5] M. Bani Issa, A. Hamoud, K. Ghadle, Giniswamy, Hybrid method for solving nonlinear Volterra-Fredholm integro-differential equations, J. Math. Comput. Sci. 7 (4) (2017) 625-641. 
[6] A. Hamoud, A. Azeez, K. Ghadle, A study of some iterative methods for solving fuzzy Volterra-Fredholm integral equations, Indonesian J. Elec. Eng. \& Comp. Sci. 11 (3) (2018) 1228-1235.

[7] A. Hamoud, K. Ghadle, On the numerical solution of nonlinear VolterraFredholm integral equations by variational iteration method, Int. J. Adv. Sci. Tech. Research 3 (2016) 45-51.

[8] A. Hamoud, K. Ghadle, The reliable modified of Laplace Adomian decomposition method to solve nonlinear interval Volterra-Fredholm integral equations, Korean J. Math. 25 (3) (2017) 323-334.

[9] A. Hamoud, K. Ghadle, The combined modified Laplace with Adomian decomposition method for solving the nonlinear Volterra-Fredholm integro-differential equations, J. Korean Soc. Ind. Appl. Math. 21 (2017) 17-28.

[10] A. Hamoud, K. Ghadle, Modified Adomian decomposition method for solving fuzzy Volterra-Fredholm integral equations, J. Indian Math. Soc. 85 (1-2) (2018) 52-69.

[11] A. Hamoud, K. Ghadle, M. Bani Issa, Giniswamy, Existence and uniqueness theorems for fractional Volterra-Fredholm integro-differential equations, Int. J. Appl. Math. 31 (3) (2018) 333-348.

[12] A. Hamoud, K. Ghadle, Existence and uniqueness of the solution for VolterraFredholm integro-differential equations, Journal of Siberian Federal University. Mathematics \& Physics 11 (6) (2018) 1-10.

[13] V. Lakshmikantham, Theory of fractional functional differential equations, Nonlinear Analysis: Theory, Methods and Appl. 69 (10) (2008) 3337-3343.

[14] S. Liao, The proposed homotopy analysis technique for the solution of nonlinear problems, Ph.D. thesis, Shanghai Jiao Tong University, 1992.

[15] S. Liao, Beyond Perturbation: Introduction to the Homotopy Analysis Method, Chapman and Hall/CRC Press, Boca Raton, 2003.

[16] S. Liao, Homotopy Analysis Method in Nonlinear Differential Equations, Beijing: Higher education press, 2012.

[17] X. Ma, C. Huang, Numerical solution of fractional integro-differential equations by a hybrid collocation method, Appl. Math. Comput. 219 (12) (2013) 6750-6760.

[18] R. Mittal, R. Nigam, Solution of fractional integro-differential equations by Adomian decomposition method, Int. J. Appl. Math. Mech. 4 (2) (2008) 87-94.

[19] S. Samko, A. Kilbas, O. Marichev, Fractional Integrals and Derivatives, Theory and Applications, Gordon and Breach, Yverdon, 1993. 
[20] C. Yang, J. Hou, Numerical solution of integro-differential equations of fractional order by Laplace decomposition method, WSEAS Trans. Math. 12 (12) (2013) 1173-1180.

[21] X. Zhang, B. Tang, Y. He, Homotopy analysis method for higher-order fractional integro-differential equations, Comput. Math. Appl. 62 (8) (2011) 3194-3203.

[22] Y. Zhou, Basic Theory of Fractional Differential Equations, Singapore: World Scientific 6, 2014.

[23] M. Zurigat, S. Momani, A. Alawneh, Homotopy analysis method for systems of fractional integro-differential equations, Neur. Parallel Sci. Comput. 17 (2009) 169-186.

DOI: $10.7862 /$ rf.2018.9

\author{
Ahmed A. Hamoud* \\ email: drahmed985@yahoo.com \\ ORCID: 0000-0002-8877-7337 \\ Department of Mathematics \\ Dr. Babasaheb Ambedkar Marathwada University \\ Aurangabad-431 004 \\ INDIA \\ Department of Mathematics \\ Taiz University \\ Taiz \\ YEMEN \\ (* Corresponding author)
}

\title{
M.Sh. Bani Issa
}

email: moh.smarh@yahoo.com

ORCID: 0000-0001-5388-9331

Department of Mathematics

P.E.T. Research Foundation Mandya

University of Mysore

570401

INDIA

Kirtiwant P. Ghadle

email: drkp.ghadle@gmail.com

ORCID: 0000-0003-3205-5498

Department of Mathematics

Dr. Babasaheb Ambedkar Marathwada University

Aurangabad-431 004

INDIA 
Mohammed Abdulghani

email: mhmed.ghnee@yahoo.com

ORCID: 0000-0003-0113-2649

Ministry of Education

Directorate General of Education Ninawa

IRAQ

Received 07.04.2018

Accepted 29.10.2018 